\title{
Operational and Fairness Issues with Connection-less Traffic over IEEE802.11b
}

\author{
Theo Pagtzis, Peter Kirstein and Steve Hailes \\ Department of Computer Science \\ University College London \\ Gower Str. WC1E 6BT \\ London, U.K. \\ \{t.pagtzis,p.kirstein,s.hailes\}@cs.ucl.ac.uk
}

\begin{abstract}
The IEEE802.11 group has recently ratified high rate (HR) extensions to enable high speed wireless communications over WLANs. 'The HR extensions specified in revision IEEE802.11b, encompass mainly new RF modulation schemes. This paper attempts an experimental evaluation of the performance characteristics of $\mathbf{8 0 2 . 1 1 b}$ in terms of throughput and loss over high speed transmission rates with respect to connection-less network traffic. We present a simple analysis of the protocol's throughput capacity over high speed rates while we reveal fundamental design con-

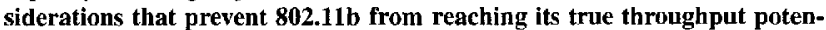
tials in the light of rate adaptivity. We further recommend some extensions to the Medium Access Control (MAC) protocol sub-layer that reconsider the multi-rate compatibility requirement while maintaining fairness in throughput between nodes at short or long distances within range from a Base Station (BS). The recommendations subject to simulations as work in-progress, expect to effect improvements in throughput over a proportionally-fair rate fallback scheme, in the order of $15 \%$ for transmission rates of $11 \mathrm{Mbps}$.We also provide some key observations to enable efficient protocol design for adaptive mobile environments.

Keywords - 802.11b, Medium Access Control, Point Coordination Function (PCF), Distributed Coordination Function DCF), Mobility, Dynamic Rate Fallback, Fairness.
\end{abstract}

\section{INTRODUCTION}

Wireless Local Area Networks (WLANs) are becoming a significant part of the Internet constituency as a popular alternative to high installation and maintenance costs incurred over wired LAN infrastructures. The license de-regulation of the ISM band has allowed WLANs to facilitate ubiquitous communication, coupled by location independent end-to-end network services in restricted spatial domains such as military settings as well as campuses, offices or enterprise facilities.

However, integrating WLANs in the Internet is not quite transparent as expected in contrast to their wire-line counterparts. Limited bandwidth availability and higher error rates induce a noticeable presence of wireless to the mobile Internet user. The long-enjoyed assumption of wire-line networks that attributes losses primarily to congestion [1], as physical medium bit error rates (BER) lie in the region of $10 \mathrm{E}-9$, is rendered in wireless networks partially invalid.

The nature of the physical medium itself, accounts for breaking this fundamental assumption. While each network segment in a LAN is electromagnetically isolated from the rest, neighboring segments in a Wireless LAN (WLAN) are prone to interference from each other. The inherent property of radio networks [2], mobility, accentuates further the problem; propagation and

This work was supported by the PIMMS research initiative, funded by the British EPSRC research council. attenuation effects, like path loss and multi-path fading are incurred as a result of varying mobility characteristics such as velocity and distance with respect to the associating Base Station. Wireless BER of the order of 10E-3 to 10E-6 [3], [4] dictate requirements for corruption control in addition to congestion control, in view of the restricted bandwidth resources available for service provisioning in diverse application domains like voice and interactive multimedia [5]. We, thus, deem essential that integrating WLAN technologies with the Internet requires a concrete understanding of the underlying medium access control (MAC) protocols deployed over real WLAN vendor implementations.

There has been a significant interest in the design [6], [7], [8], and standardization [9], [10] of local area communication protocols for wireless networks. Amongst them the study group 802.11 formed under IEEE802 project, and the ETSI High Performance European Radio LAN (HIPERLAN) [11], [12], [13] both commissioned to provide recommendations for an interoperable WLAN standard. Both standards target the physical (PHY) and medium access control sub-layer. A number of vendor implementation have adopted the 802.11 standard while recently the IEEE802.11 study group ratified high rate (HR) extensions over the protocol standard to effect higher transmission rates.

This work concentrates on experimental evaluation of the $802.11 \mathrm{~b}$ protocol [14]. We investigate the performance profile of 802.11 b over proven vendor implementations. This work also aims in verifying and extending results in [15], [16], [17] over the new HR extensions. For the sake of consiceness, the paper presents conclusive summaries of our findings; these assume a basic understanding of the DCF and PCF functions of 802.11b. For more comprehensive discussions and results, the interested reader is invited to refer to [18] or for detailed background information, the IEEE 802.11 specification [14].

This paper is organized as follows: Section II details the environment and methodology of our experimental setup. Section III presents results over the experimental scenarios as well as transmission overheads accounted for the MAC and PHY layers of $802.11 \mathrm{~b}$. Section IV presents fairness issues with regard to rate compatibility for $802.11 \mathrm{~b}$. We conclude with a summary of our findings in Section V. 


\section{ENVIRONMENT AND METHOdOlogy}

The experiments employed the Lucent IEEE/802.11b wireless network interfaces ${ }^{1}$, operating at $2.4 \mathrm{GHz}$ over Direct Sequence Spread Spectrum (DSSS) and delivering signaling rates up to $11 \mathrm{Mbps}$.

To the best of authors' knowledge, most of the published results ${ }^{2}$ have been considering first generation (WAVELAN I) network interfaces; these have been 802.11 implementations employing low bandwidth RF modulation schemes with maximum signaling rates up to 2 Mbps. To this end, this paper aims to contribute fresh observations about the protocol's performance over it's HR extensions.

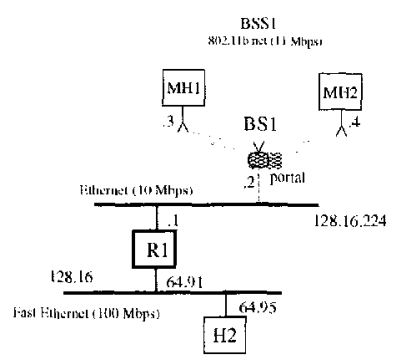

Fig. 1. Experimental environment employed

The experimental topology of Figure 1 featured a set of four FreeBSD (4.1), Pentium class, PC hosts. Both MH1 and MH2 were equipped with PCCARD $802.1 \mathrm{lb}$ WNICs. These were driven by the device drivers included in the above OS release. On the Ethernet side H1 and R1 featured Fast Ethernet network interfaces. The Access Point BS, was an $802.11 \mathrm{~b}$ (L2) bridge available from the same vendor; its wired segment featured a $10 \mathrm{Mbps}$ Ethernet interface ${ }^{3}$. A PC-based Access Point was not considered for reasons of efficiency and performance described in [18]. The private wireless subnet routed by $\mathrm{R} 1$ ensured no bypassing traffic during the course of the experiments.

For the generation of traffic patterns we engineered TrafSim [18]. Through TrafSim, the tests featured variable syncronisation over configured client permutation test sets, variable isochronous transmission rates and signal strength tracing in unattended mode. Our traffic patterns generation borrowed implementation ideas from other well-known measurement tools like rtptools [19] and DBS [20].

We increased the clock frequency of the FreeBSD kernel to $1000 \mathrm{~Hz}^{4}$ effecting isochronous transmission rates up to 12 Mbps. This would be sufficient for simulating congestion conditions over the $802.11 \mathrm{~b}$ link. We ensured that increasing the kernel clock resolution bears no adverse effects to the performnace of the OS over the available set of processor speeds [18]. Test packet flows consisted of $10 \mathrm{~K}$ packets, while test permutations generated by the (send rate, packet size) test space tuple, were repeated 15 times to ensure reduced variance in observed values.

\footnotetext{
1 referred as WNICs henceforth

${ }^{2}$ to the date of writing

${ }^{3}$ there was no Fast Ethernet interface on any Access Point implementation at the time of writing

${ }^{4}$ default value is $100 \mathrm{~Hz}$
}

Experiments were isolated ${ }^{5}$ from potential sources of radio interference with SNR readings captured throughout each test on the WNIC. Post-measurement processing and analysis was carried out on traffic traces captured near the receiver. All tests were performed in both directions between Ethernet and $802.11 \mathrm{~h}$ peers. Our results were tested against a confidence interval of $95 \%$ to ensure statistical validity.

\section{EXPERIMENTAL SCENARIOS}

\section{A. Peak Throughput and Error Rate}

Performance of the $802.1 \mathrm{lb}$ WNICs in terms of peak throughput and error rates was first tested with respect to wireline LAN segment. The wireless host, MH1 was stationary with very small distance from the BS1. Figure 2 shows, the user throughput at the wireless receiver reaching a maximum of $6.22 \mathrm{Mbps}$ (528.91 packets $/ \mathrm{sec}$ ) for packet sizes close to the MTU $\mathrm{M}^{6}$. This is $56.36 \%$ of the total bandwidth capacity of the $11 \mathrm{Mbps} 802.1 \mathrm{lb}$ link. $44 \%$ of the signaling rate is consumed on transmission overheads and latencies.

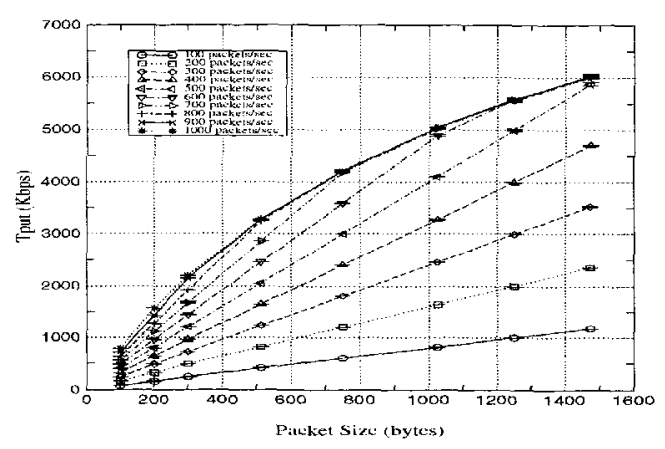

Fig. 2. Throughput on Wireless receiver (varying packet size)

Throughput performance over different send rates exhibits a somewhat different behavior. This is shown in Figure 3. It can be seen that above a certain send rate threshold, when packet size remains constant, user throughput remains constant also. This may seem counter-intuitive when compared against throughput performance of Fig.2. However, it is clearly explained by looking at the corresponding frame error rates for constant throughput when the send rate increases, as well as considering that the Ethernet link supported by BS is $10 \mathrm{Mbps}$.

From the throughput peaks of Figure 3 and the send rate points of Figure 5, we deduce that increasing the send rate beyond a certain threshold, for constant packet size, saturates the $10 \mathrm{Mbps}$ Ethernet segment between R1 and BS. This implies at the points where the throughput remains constant, BS exhausts its receive buffers on its $10 \mathrm{Mbps}$ Ethernet interface as it receives packets at a rate faster than its $10 \mathrm{Mbps}$ link can cope with, during reception. As such, the wired segment of BS will drop packets before these ean be transmitted over the wireless segment. In the reverse direction throughput shows identical behaviour for send rates up to $500 \mathrm{pps}$ (and thus omitted from the graph), while it drops with higher variance at offer loads of

\footnotetext{
${ }^{5}$ conducted during the night

${ }^{6}$ assumed to be 1500 bytes
} 
$600 \mathrm{pps}$ or above. Such behaviour may be attributed to both the driver efficiency the WNIC in MH1 as well as the limitation imposed by the $10 \mathrm{Mbps}$ Ethernet link effected by the BS.

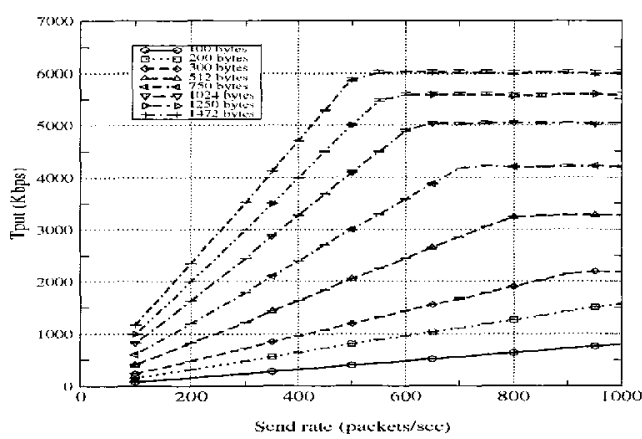

Fig. 3. Throughput on Wireless receiver (varying send rate)

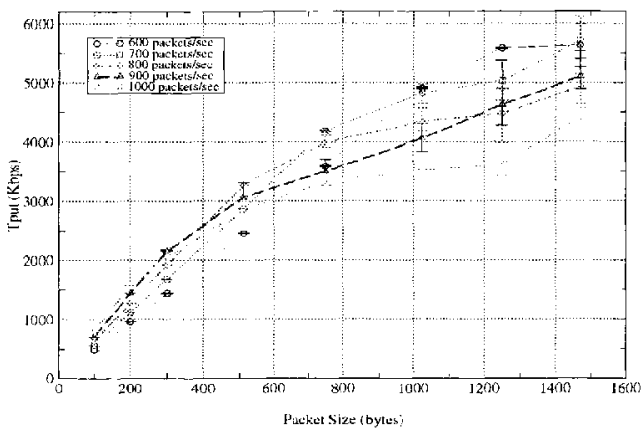

Fig. 4. Wired receiver throughput (varying packet size)

With respect to packet loss rate, Figure 5 shows that for transmission rates up to $550 \mathrm{pps}$ frame error rates (FER) are in the region of $4 \mathrm{E}-3$, implying that at peak rates 1 in 250 frames on average is lost for packet sizes close to the Ethernet MTU. Beyond 550 pps the FER become significantly high (way above $1 \%$ ) implying a saturated link beyond the transmission capacity of the protocol.

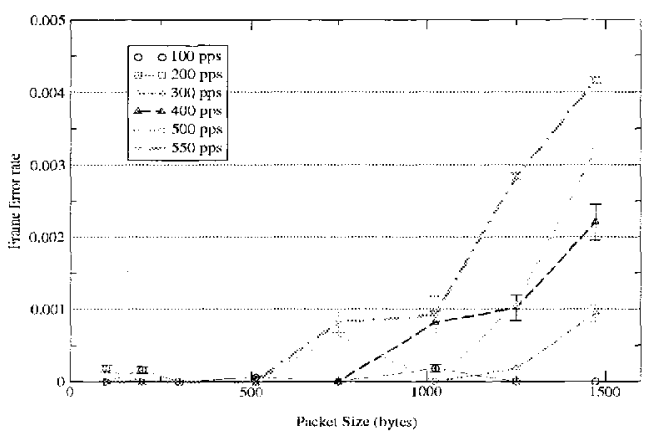

Fig. 5. Packet Loss Rate of Wireless receiver

On the wired receiver, the throughput becomes noisy and effectively oscillates for send rates above $600 \mathrm{pps}$, while the max- imum user throughput is observed at $526.36 \mathrm{pps}(6.19 \mathrm{Mbps})$ for packet size of 1500 bytes. Loss rate curves on the wired receiver are similar to Figure 5 and, thus, omitted. However, in this case it is the wircless sender that experiences buffer overflows ${ }^{7}$ as transmission rates above 550 pps overrun the transmission buffer capacity of the wireless interface. This implies that the output queue if_output () of the wireless interface gradually fills up to the point where the transmission buffers of the wireless interface are exhausted and frames in the output queue get dropped.

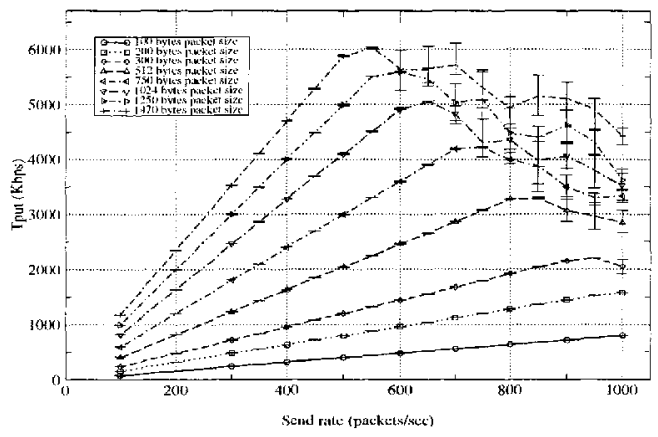

Fig. 6. Wired receiver throughput (varying send rate)

\section{A.1 Transmission Overheads}

In 802.11 DCF/PCF function, transmission time is allocated according to the CFP repetition interval $T_{C F P_{r i}}$ and is equal to:

$$
T_{C F P_{r i}}=T_{C P}+T_{C F P}
$$

where $T_{C P}$ is the time allocated to DCF (contention period) and $T_{C F P}$ the time allocated to PCF (contention-free pollable period).

\section{A.2 DC Function Overheads}

In contention-based (CP) mode, the wireless node has to (re)contend for the medium for every $\mathrm{MPDU}^{8}$ scheduled for transmission $(\mathrm{Tx})$. The $\mathrm{Tx}$ cost of a single MPDU during $\mathrm{CP}$ mode (including the cost induced by the binary exponential backoff, as the contention window size), assuming RTS/CTS handshake is:

$$
\begin{aligned}
C_{C P_{T x d a t a}=} & C_{d i f s}+3 C_{s i f s}+C_{R T S}+C_{C T S} \\
& +C_{M P D U_{d a t a}}+C_{A C K}+C_{B a c k o f f} \\
C_{\text {Backoff }}= & \operatorname{int}\left(C_{w i n} \cdot \operatorname{rnd}()\right) \cdot T_{\text {slot }} \\
C_{\text {win }}= & 2^{2+\rho}-1, \wedge r n d() \epsilon[0,1], \rho \in \mathbb{N} \wedge \rho \in[1,6]
\end{aligned}
$$

with $r n d()$ the pseudo-random number generator and $C_{w i n}$ the contention window size randomized. $\rho$ is the number of attempts to capture the medium after the expiry of the backoff counter and a subsequent collision with another node that attempts to do

\footnotetext{
${ }^{7}$ the output queue is typically 50 packets long

${ }^{8}$ in the 802 .X terminology a MAC protocol data unit (MPDU), is the unit that encapsulated the data payload
} 
so. $T_{\text {slot }}$ has a nominal value of $20 \mu \mathrm{sec}$ and identifies a unit measure of $C_{w i n}$. The $C_{w i n}$ value increases exponentially on a re-capture attempt of the medium following a collision, with a freeze after six consecutive collisions. $C_{d i f s}$ and $C_{s i f s}$ represents inter-frame intervals that account for Tx latencics such as propagation and $\mathrm{Rx} / \mathrm{Tx}$ turnaround times. Table I lists the header cost at different contention modes and layers of both encapsulating and encapsulated framing. It can be seen that control and framing overheads total to 178 bytes for Tx of an MPDU during CP. For MPDU payload below the RTS_Threshold, the RTS/CTS mechanism is not employed; that brings the Tx cost down to 96 bytes of framing for both MAC and PHY.

TABLE I

FRAME TYPES ANI ASSOCLATED HEADER IIINGTH

\begin{tabular}{|c|c|c|}
\hline Mode & frame type & $\begin{array}{c}\text { length } \\
\text { (bytes) }\end{array}$ \\
\hline any & PLCP - PHY & 24 \\
\hline CP & MSDU (RTS) - MAC & 20 \\
\hline CP & MSDU (CTS) - MAC & 14 \\
\hline CP & MSDU (ACK) - MAC & 14 \\
\hline any & MPDU - MAC & 34 \\
\hline CFP & $\begin{array}{c}\text { MMPDU (Beacon) - } \\
\text { MAC }\end{array}$ & $33-323$ \\
\hline CFP & MSDU (CFend) - MAC & 20 \\
\hline CFP & $\begin{array}{r}\text { MPDU } \\
\text { CFpoll,ack,poll+ack - } \\
\end{array}$ & 34 \\
& MAC & \\
\hline
\end{tabular}

\section{A.3 PC Function Overheads}

We are interested in the lower bound of transmission costs effected by a single host. Such bound is described for both uni-/bidirectional traffic as the initialization time of CFP and a single data frame exchange between the AP and the node separated by the respective IFS intervals. This is:

$$
\begin{aligned}
C_{C F P_{\text {Txdata }}=}= & C_{C F P_{i n i t}}+C_{M D P U_{\text {poll }}}+ \\
& C_{M P D U_{D 10 u t+a c k}}+ \\
& C_{M P D U_{D 2 i n+a c k}}+2 C_{\text {sifs }} \\
C_{C F P_{\text {init }}}= & C_{p i f s}+C_{\text {beacon }}
\end{aligned}
$$

Table II gives a summary of header overheads per user data packet. Both PCF and DCF functions incur similar transmission overheads if RTS/CTS is employed. Exception to this is the setup cost for PCF which iterates per CFP repetition interval. This implies that a longer CFP amortizes the setup cost over more MPDUs in either direction.

\section{A.4 High Rates and Signaling Time Cost}

The 64-code set [21] at the $11 \mathrm{Mbps}$ signaling rate, allows a total of 8 bits of information to be encoded per CCK symbol [22]. At $5.5 \mathrm{Mbps}$ the processing reduces the amount of information bits per codeword to 4 . Both 5.5 and $11 \mathrm{Mbps}$ rate support a codeword transmission rate of $1.375 \mathrm{MHz}$ as the PN-sequence is now 8 chips instead of 11 supported in 1 and 2
Mbps. As such, each bit is Tx-ed at $11 \mathrm{Mbps}$ between a wireless node and the Base Station in $90.909 \mathrm{nsec}$

Furthermore, depending on the coordination mode (DCF or $\mathrm{PCF}$ ), the per MPDU Tx $\operatorname{cost}^{9}$ comprizes of the following constituent components:

Tx time of MAC frame payload. The size of the frame body may vary between $0-2312$ bytes. Subsequently, the time slot required for transmitting such payload $C_{\text {pload }}$ varies in the interval of $[0,1681.44] \mu \mathrm{sec}$.

CP-mode Tx with RTS/CTS. The cost of a single MPDU transmission (dominated by $C_{w i n} \in[0,255]$ ) becomes:

$$
907.62+C_{\text {pload }} \leq C_{C P_{\text {Txdata }}} \leq 6007.62+C_{\text {pload }} \mu \text { sec }
$$

CP-mode Tx without RTS/CTS. A case for multicast/broadcast data frames or for MPDU frames below the RTS threshold; an ACK is only required in the case of unicast MPDUs transmitted during CP. This yields:

$$
478.9+C_{\text {pload }} \leq C_{C P_{\text {Tadata }}} \leq 5708+C_{\text {pload }} \mu \mathrm{sec}
$$

During the experiments $C_{p l o a d}$ varied between 146 and 1518 bytes (including Ethernet headers). That is, the lower time bound $\left(C_{w i n}=0\right)$ required for a successful packet transmission is in $[585.08,1581.9] \mu \mathrm{sec}$.

CFP-mode transmission (PCF). This encompasses the CFP initialization time prior to any time spent in actual data transmissions. Depending on the size of the Beacon frame, the cost is bound by $[236.36,447.27] \mu$ sec. The beacon size and, thus, Tx time depends to a small extent on the size of the Service Set ID (SSID) ${ }^{10}$. It's main dependency is the size of the traffic indicator map (TIM) ${ }^{11}$, which indicates outstanding traffic for delivery to stations. As the CF period commences after PIFS time, the total $C_{C F P_{\text {in it }}}$ cost increases by $30 \mu \mathrm{sec}$ at the bounds of the above interval. Thus, $C_{C F P_{\text {Txdata }}}$, as in (3), for a single station during unidirectional CFP transmission of an MPDU is $680.16+C_{\text {pload }} \mu$ sec.

In the presence of $\mathrm{N}$ stations, the cost of $C_{C F P_{\text {init }}}$ is spread amongst them, becoming:

$$
\begin{aligned}
& \frac{266.36+680.16 \dot{N}}{N}+C_{\text {pload }} \leq \\
& \leq C_{C F P_{\text {Txdata }}} \leq C_{\text {pload }}+\frac{477.27+680.16 \dot{N}}{N}
\end{aligned}
$$

${ }^{9}$ Details on the individual figures can be found in [18]

${ }^{10}$ that may vary between $2-34$ bytes

${ }^{11}$ TIM varies hetween $1-251$ bytes

TABLE II

CONTENTION MODES AND ASSOCIATFD OVERHEAISS

\begin{tabular}{|c|c|}
\hline Mode & $\begin{array}{c}\text { header o/heads } \\
\text { (bytes/MPDU) }\end{array}$ \\
\hline$C P_{R T S / C T S}$ & 178 \\
\hline $\mathrm{CP}$ & 96 \\
\hline$C P_{\text {init }}$ & $\mathrm{n} / \mathrm{a}$ \\
\hline $\mathrm{CFP}$ & 174 \\
\hline$C F P_{\text {init }}$ & $57-347$ \\
\hline
\end{tabular}


For bidirectional traffic, the Tx cost of a single MPDU increases by an extra data frame of potentially different size. For cach frame sent by the wireless host, the AP is expected to Tx a frame pending for delivery to that station. That multiplies $C_{\text {pload }}$ by 2 for bidirectional traffic. The above figures assume that $\mathrm{CF}-$ Polls are not lost and that stations ACK the poll message. At this stage they do not include the case where a node does not respond to the CF-Poll. This is described in [18].

Figure 7 shows how $\mathrm{Tx}$ cost increases with the MPDU payload during DCF and PCF, for a single station associations with the AP. It can be seen that PCF with a single wireless source incurs almost the same cost as DCF with RTS/CTS enabled for the entire size range of an MPDU payload, when the contention window lies between counter values of a single backoff attempt (i.e. no collisions). This is not the case for the basic DCF access mode with no RTS/CTS control frames. Such observation doesn't hold if the number of stations associated with the AP is $N>1$.

Transmission times are considerably shorter when no RTS/CTS access control is enabled. However, this is true only for a small number of stations and with small offered load (low contention). As the number of associated nodes together with their offered load grows, contention for medium capture increases; this induces a higher probability of collisions [23] which throttles the contention window and number of Backoff attempts. This is the primary cause for growing transfer delays during DCF as shown by Chhaya [24] and Chen [25] which may be reduced through the use of RTS/CTS control. The latter is, however, not a panacea as it renders beneficial only above a certain frame length [26].

We further evaluated the validity of our results with respect to throughput and loss by approximating numericaly the protocol's capacity under both PCF and DCF [18]. The estimate approximation of the transmission capacity was found somewhat higher (around $6.54 \mathrm{Mbps}$ ) than observed in the throughput results. Since we did not experience collisions for a single station (since the AP does not deliver any packet, while ACKs arrive in response to transmissions and before the renewal period set by the next DIFS interval) we concluded that such difference is largely due to inefficiencies of the driver.

DCF offers a fairer chance of acquiring the medium by having all nodes contending for channel access after each MPDU transmission. However, the probability of collisions during a backoff is bound to increase with the number of stations associating with the AP; this can increase the backoff time for a single node up to $5.3 \mathrm{~ms}$. As shown in (4), $C_{\text {win }}$ is dominant during an MPDU transmission over DCF and can, thus, induce significant variance per frame payload (packet). This reasons for DCF being unable to offer distributed time-bounded services (DTBS). On the contrary, PCF, through its CF polling scheme sets specific upper bounds of delay.

Measurements showed that user throughput can increase up to $7.8 \mathrm{Mbps}$ for packet sizes larger than 1500 bytes and up to 2266 bytes. This figure is, however, non-realistic considering that packet size mostly bound by the MTU size; the standard MTU can be as low as $\mathbf{5 8 6}$ bytes, while specific application classes, such as interactive multimedia or VoIP, strive for small packet sizes. For instance, audio packets range between $50-500$ bytes depending on the codec and the error resilience scheme employed. Video packets peak around 1024 bytes. It is, thus, clear that a MAC protocol should strive to increase efficiency and capacity over small packet sizes rather than emphasizing on large packet size for protocol capacity.

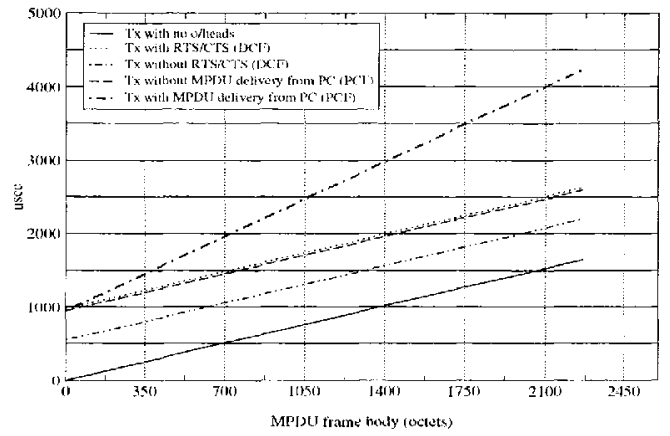

Fig. 7. Transmission cost of single data frame under DCF and PCF

\section{B. Bi-directional streaming over the wireless link}

This scenario looked at MAC behaviour during simultaneous transmission and reception. The tests simulated overload conditions over send rates of up to $400 \mathrm{pps}(4.7 \mathrm{Mbps})$ in each direction. Measurements were taken only during times that the WNIC was interleaving between both $\mathrm{Tx}$ and $\mathrm{Rx}$ of streamed UDP flows. In this manner the throughput and loss results were prevented from being inflated by single transmission fragments if one of the two peers finished its Tx earlier.

TABLE III

BI-DIRFCTIONAI. THROUGHIUT AT SIGINIFICANCE LFVHI. 0.95

\begin{tabular}{|c|c|c|c|c|c|c|}
\hline \multirow{2}{*}{$\begin{array}{c}\text { Packet } \\
\text { Size } \\
\text { (bytes) }\end{array}$} & \multicolumn{6}{|c|}{ Throughput (Kbps) } \\
\cline { 2 - 7 } & \multicolumn{2}{|c|}{$200 \mathrm{pps}$} & \multicolumn{2}{|c|}{$300 \mathrm{pps}$} & \multicolumn{2}{|c|}{$400 \mathrm{pps}$} \\
\hline \hline 150 & 221 & 240 & 316 & 360 & 411 & 479 \\
\hline 250 & 367 & 400 & 527 & 600 & 689 & 799 \\
\hline 350 & 516 & 560 & 739 & 840 & 947 & 1120 \\
\hline 450 & 670 & 720 & 949 & 1080 & 1213 & 1439 \\
\hline 550 & 807 & 880 & 1139 & 1320 & 1470 & 1759 \\
\hline 650 & 953 & 1040 & 1338 & 1560 & 1723 & 2077 \\
\hline 750 & 1091 & 1200 & 1543 & 1800 & 1941 & 2397 \\
\hline 950 & 1365 & 1520 & 1945 & 2280 & 2476 & 2689 \\
\hline 1050 & 1497 & 1680 & 2098 & 2520 & 2722 & 2711 \\
\hline 1250 & 1786 & 1999 & 2413 & 2998 & 3001 & 2900 \\
\hline 1350 & 1921 & 2161 & 2569 & 3234 & 3097 & 2995 \\
\hline 1470 & 2026 & 2353 & 2785 & 3470 & 3230 & 3073 \\
\hline
\end{tabular}

We observed that for Tx rates up to $300 \mathrm{pps}(3.52 \mathrm{Mbps})$ in each direction, the MH2 receiver experienced higher throughput than the $\mathrm{H} 2$ receiver. While for small packet sizes the increase in the observed throughput values at the 802.11 station was only marginal, as packet size grows the increase gets slightly bigger (see Fig. III). This was true for all packet sizes up to 1470 byles of user data, even though the wireless link was running into mild 
congestion at a combined send rate of $7.05 \mathrm{Mbps}$. The above is independent of which host initiated Tx first.

The higher throughput experienced at the wireless receiver largely justifies the original obscrvation that the wired source would complete transmission faster than the wireless host. While a detailed discussion is provided in [18] we inferred from the sequence numbers of frames exchanged between BS1 and the $802.11 \mathrm{~b}$ station, that the latter is a slow sender compared to BS1. This can only be attributed to different implementations between the FreeBSD WNIC driver and the kernel firmware of BS1. This make clear that the performance of the driver induces its own limitations over representative inference about the protocol's performance.

While the 802.11 receiver experienced higher throughput than the fixed receiver, it also witnessed higher loss compared to the fixed host. In fact, it is suprizing to note that the fixed receiver did not experience any loss at all even for send rates up to $400 \mathrm{pps}$ in each direction. On the contrary FER at the 802.11 station varied between $8 \mathrm{E}-4$ and $8 \mathrm{E}-3$ for packet sizes above 1150 bytes and 300 pps send rate. This is shown in table IV. For lower send rates ( 100 and $200 \mathrm{pps}$ ) we observed only single burst losses that varied between 1-6 packets. Longer loss runs (6-10 packets) were observed but were extremely sparse (1.25E-05) for aggregate send rates below the observed peak throughput of the $802.11 \mathrm{~b}$ link.

TABLE IV

LOSS RATE ON HIGH-THROUGHPUT WIRFI FSS RECFIVER

\begin{tabular}{|c|c|c|}
\hline Packet Size & Loss Rate (@ 300pps) & CI 95\% \\
\hline \hline 1150 & $8.61 \mathrm{E}-4$ & $1.33 \mathrm{E}-4$ \\
\hline 1250 & $5.29 \mathrm{E}-3$ & $8.13 \mathrm{E}-4$ \\
\hline 1350 & $2.12 \mathrm{E}-3$ & $4.26 \mathrm{E}-4$ \\
\hline 1470 & $8.36 \mathrm{E}-3$ & $1.84 \mathrm{E}-4$ \\
\hline
\end{tabular}

\section{Throughput fairness between different wireless hosts}

Fairness tests considered identical offered loads between two $802.11 \mathrm{~b}$ hosts ( $\mathrm{MH} 1$ and $\mathrm{MH} 2$ ), that transmit to a single wired host from initially stationary positions and a distance less than 2 meters from BS1. Transmission rate and packet size were kept constant and within the capacity of the MAC protocol (up to 300 pps) for each wireless sender.

Figure 8 shows the reduction in throughput ${ }^{12}$, when at time $T$ $\mathrm{MH} 2$ attempts to send traffic during an in-progress transmission by MH1. As expected, there was no significant difference in the observed throughput of these hosts. The average throughput for the two $802.11 \mathrm{~b}$ hosts, was $3.15 \mathrm{Mbps}$ and $3.17 \mathrm{Mbps}$ respectively. This implies that for small number of $802.11 \mathrm{~b}$ nodes the MAC sublayer allocates a fair share of its bandwidth capacity for stations with similar distances from the base station. In addition, the MAC layer offered the same aggregate throughput irrespective of the number of associated $802.11 \mathrm{~b}$ stations (peak of $6.22 \mathrm{Mbps}$ ).

Distance of MH1 from BS1 was then increased, while the distance of transmitter MH2 was kept constant. As shown in Fig-

\footnotetext{
${ }^{12}$ throughput of the incoming transmission is shifted along the $X$ axis so as to observe potential differences
}

ure 9 , bandwidth sharing between the two $802.11 \mathrm{~b}$ hosts at different distances from BS1 was no more the same. This is not unreasonable since the propagation delay increases with no increase in the transmitter's power. Propagation effects instigate poor channel conditions over distance. This induces increased corruption in the received data or control or management frames which triggers retransmissions through ARQ. Without ARQ at this stage, corruption in received frames escalates to subsequent collisions which if sustained at high signaling rates, may render the MAC protocol unstable. Thus, during poor channel conditions, retransmissions use up the bandwidth and thus reduce the effective data rate ${ }^{13}$.

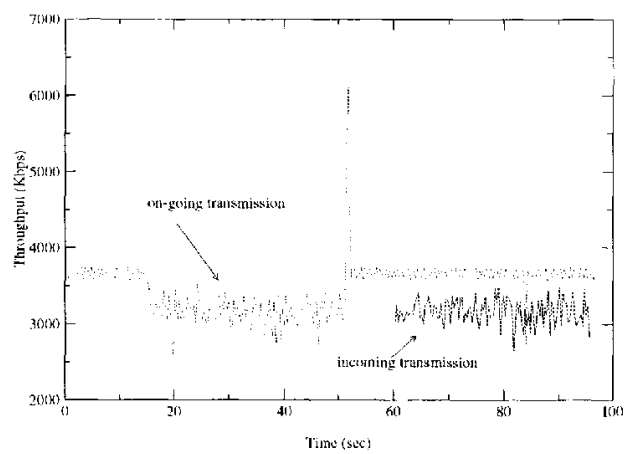

Fig. 8. Throughput for two simultaneous transmitters with symmetric distances of 2 meters

From the above one can now justify the observed drop in throughput for the on-going transmission of Figure 9. That, may be erroneously interpreted as lack of fairness in the throughput experienced by two stations at different distances, but within range from the AP. We observe that the measure of distance of the wireless host from the Base Station behaves naturally as a service differentiator. The farther a wireless node moves away from its Access Point, the more its bandwidth and subsequently throughput is bound to degrade. Of course the measure of distance is based explicitly on the measure of the underlying Signal to Noise Ratio (SNR) that the wireless node is experiencing.

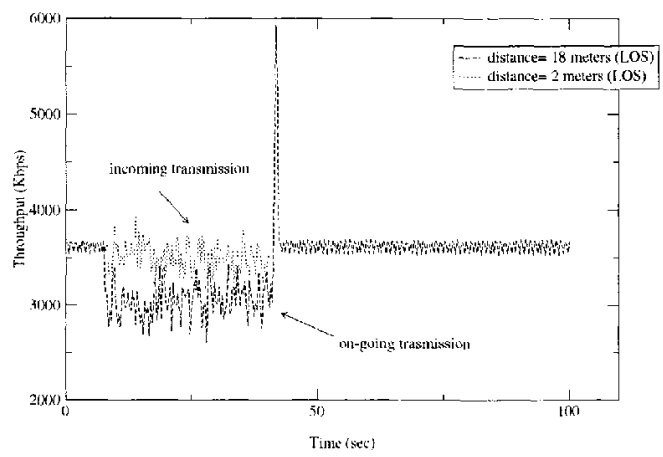

Fig. 9. Throughput for two simultaneous transmitters with assymetric distances of 18 meters

${ }^{13}$ the effective data rate may be defined a the rate of the information that is correctly transmitted 
We, thus, argue that in the face of differentiated services over wireless networks, a provisioning model must consider some measure of proportional fairness in bandwidth allocation, where bandwidth may be traded-off with certain SNR levels to maintain connectivity. The SNR, however, may also be affected by other factor orthogonal to distance, such obstructions in the line of sight, or bypassing interference. Nevertheless, in all of these case the measure of SNR may discribe some measure of mobility for the wireless host. Furthermore we argue the measure of SNR should also act as a link adaptation notification signal that need be propagated to the IP layer so as to allow faster $\mathrm{L} 3$ response times in the face bandwidth fluctuation.

\section{RATE COMPATIBILITY AND FAIRNESS}

A basic requirement in the selection of the high-rate modulation scheme has been inter-operability with the base 802.11 protocol. For that purpose, the signal acquisition sequence (PLCP) of $802.11 \mathrm{~b}$ was designed to be tranceived ${ }^{14}$ at $1 \mathrm{Mbps}$ across all supported rates, while sending its frame payload at different rates. As such, any 802.11 b station can sense and defer ${ }^{15}$ access to the medium if they cannot process frames at these rates. Table $\mathrm{V}$ shows the calculated transmission times/bit for all $\mathrm{Tx}$ rates supported by the $802.11 \mathrm{~b}$ specification.

TABLE $V$

SUPPORTED RATES AND TX TIME/BIT

\begin{tabular}{|c|c|c|c|}
\hline $\begin{array}{c}\text { rate } \\
\text { (Mbps) }\end{array}$ & $\begin{array}{c}\text { bits per } \\
\text { symbol }\end{array}$ & $\begin{array}{c}\text { symbol } \\
\text { Tx rate }\end{array}$ & $\begin{array}{c}\text { Tx } \\
\text { time/bit }\end{array}$ \\
\hline 1 & 1 & $1 \mathrm{Mhz}$ & $1 \mu$ sec \\
\hline 2 & 2 & $1 \mathrm{Mhz}$ & $500 \mathrm{nsec}$ \\
\hline 5.5 & $2+2$ & $\begin{array}{c}1.375 \\
\mathrm{Mhz}\end{array}$ & $\begin{array}{c}181.8175 \\
\mathrm{nsec}\end{array}$ \\
\hline 11 & $6+2$ & $\begin{array}{c}1.375 \\
\mathrm{Mhz}\end{array}$ & $\begin{array}{c}90.90875 \\
\mathrm{nsec}\end{array}$ \\
\hline
\end{tabular}

While this serves nicely the rate compatibility objectives, it trades off bandwidth from high signaling rates for the purpose of maintaining connectivity for entire set of associated stations. As such, the $802.11 \mathrm{~b}$ protocol sets a lowest common denominator of transmission rate for all stations associated with the AP of a single cell. This is inherently unfair for any $802.11 \mathrm{~b}$ station operating at signaling rate above 1 Mbps.

We have shown [18] that the PHY preamble and header aquisition sequence consume $192 \mu$ sec over 1 Mbps modulation. If the PHY aquisition sequence was modulated over the 2, 5.5 or 11 Mbps signaling rate, it would require only $96,34.9$ and 17.45 $\mu s e c$. This is $2,5.6$ and 11 -fold reduction in transmission cost of the PHY layer acquisition sequence! Such savings are quite significant if we consider the transmission cost of a single data frame, especially for small frame payloads over real-time traffic.

Figure 10 illustrates the cost of acquisition sequence $C_{a s}$ in proportion to the transmitted packet. $C_{a s}$ is considerably larger than the cost of the transmitted payload at $1 \mathrm{Mbps}$, especially for packet sizes up to 264 bytes. At this point $C_{a s}$ is equal to the cost of transmitting the 264-byte payload sustaining a poor

${ }^{14}$ constant Tx cost of $192 \mu \mathrm{sec}$

${ }^{15}$ NAV update utilization of the medium. Utilization, however, improves significantly if the higher transmission rates (modulation schemes) are effected over the acquisition sequence.

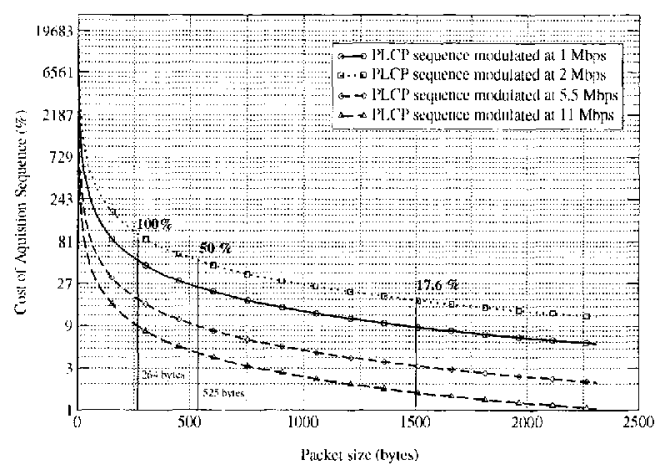

Fig. 10. Transmission cost of the PHY layer acquisition sequence in proportion to the transported payload

At the rate of $11 \mathrm{Mbps}$ the reductions rendered over the PLCP sequence would imply a minimum increase of $15.5 \%$ ( 1 in 8 frames at 1500 bytes) in the throughput rates currently experienced over $802.11 \mathrm{~b}$ at $11 \mathrm{Mbps}$. We say a 'minimum' because the figures given above do not encompass the PHY layer cost reductions on control (SPDU) and management (MMPDU) frames exchanged prior any data frame transmission.

Of course the design requirement for continuous connectivity beyond the SNR levels offered at the $11 \mathrm{Mbps}$ signaling rate could be accommodated by the straightforward solution of populating the required coverage area with more APs. This is, however, a naive solution towards the robustness of the $802.1 \mathrm{lb}$ protocol itself.

To cater for the resolution of this fairness issue over the critical requirement of rate compatibility we propose an extension to the $802.1 \mathrm{lb}$ protocol that makes use of the aforementioned observations of Section III-C. In particular, this extension scheme instigates traffic differentiation for the purpose of resolving the fairness issue. A brief description is provided in the following section.

\section{A. Resolving Fairness through Traffic Differentiation}

In this scheme we assume that each station modulates at its own operational signaling rate both for its PLCP sequence and frame payload. However, we require that the association/reassociation function, if solicited by the 802.11 station, is effected with a PLCP sequence modulated at 1 Mbps. The primary requirement for the stability of the protocol is that the stations do not attempt to contend for channel access in the event they operate at a different signaling rates from the operational one currently supported by the AP.

Our scheme proposes that on the side of the Access Point, the Point Coordinator maintains multiple PCF polling lists, instead of the single standard one. For that we define the notion of a Polling List Set (PLS) denoted as $\Psi$. Such set will comprise the set of all active polling lists and should be expandable to accommodate new ones. Each polling list $\psi_{i} \epsilon \Psi$, will map to a single element of the set $Q$, which is defined as the set of all 
supported discrete bandwidth classes. We also define the set of all supported modulation schemes in current or future versions of 802.11 as $M$. Each element $q_{i}$, may identify one or more elements $m_{i}$ from $M$. That is, a single bandwidth class may encompass a range of discrete supported bandwidths.

The union of all $\Psi_{n}$ constitutes the set of all stations $N_{A P_{m}}$ found in a single $802.11 \mathrm{~b}$ cell for that $A P_{m}$. The scheme initially considers two bandwidth classes for 802.11 b stations:

- a bandwidth class that supports associations for highest operational signaling rate (i.e. $11 \mathrm{Mbps}$ ); this is denoted as $Q_{h}$.

- an aggregate bandwidth class that supports associations for all lower supported signaling rates (such as 5.5, 2 or $1 \mathrm{Mbps}$ )

Each polling list will map naturally to a single contention-free pollable period CFP. Thus, we define further the set of all CF periods as $\Omega$. The size of both $\Psi$ and $\Omega$ sets would be dictated by the number of the stations associated with each list as well as their supported and operational signaling rates. The ordering of elements in $\Psi$ at the moment dictates a relative priority according to its bandwidth class. This implies that depending on the order high bandwidth classes may be treated first or last. That puts some extra significance on the size of the individual CF period of $\omega_{n}$, but most important the total length of all CF periods, i.e. $\sum_{i=1}^{n} \omega_{i}$. We are currently investigating optimal ordering schemes with CFP repetition intervals of around $50 \mathrm{~ms}$ for this purpose.

In set $\Omega$, for each following $\omega_{l}$ the CF-End message of $\omega_{l-1}$ will signal to the stations in $p s i_{l-1}$ the duration of $\omega_{l}$, so that for each station $S T A_{k_{\psi_{i-1}}}$ access to the medium is deferred during that period. This is effected by adjustment of the network allocation vector $N A V_{k_{\psi_{l-1}}}$ for the $k$ th station. Alternatively, and in the event that such action does not hinder the performance of stations in $\psi_{i}$, a station from $p s i_{l}$ may consider re-associating with the BS for $\psi_{n}$, where $l \neq n$ for some different bandwidth class $q_{n}$.

The 802.11 protocol is required to maintain rate compatibility for two reasons: one is the possibility that a wireless station cannot support certain HR modulation schemes; the second is that even if a station supports all available modulation schemes, the dynamic rate-shifting mechanism of 802.11 will up/downgrade its signaling rate according to its SNR levels from the AP. Both cases, however, rely on the capture of the acquisition sequence.

As such in both situations ${ }^{16}$ the station (re)associates with the Point Coordinator as a rate-limited CF-pollable station that will operate only under PCF for a particular CFP identifier. The DCF function for that polling list $p s i_{l-1}$ of associated stations is prohibited. To complement for the removal of the DCF function from that class of stations, we consider the optional resizing of the CFP period per $\psi_{i}$ to accommodate for repeated polls of stations of the low-bandwidth service class that has expressed such interest/request. This may be conveyed over standard traffic indication map (TIM) type that can be configurable not only by the BS but also by the station of that Polling list.

\section{B. Propagating the Bandwidth Differentiation at the IP layer}

Availability of different signaling rates, through either the dynamic rate-shifting mechanism of 802.11 or restricted support

\footnotetext{
${ }^{16}$ stationary or mobile within the range of the AP
}

for high-rate modulation schemes by stations effect a change in link's characteristics without notification to the IP layer and subsequently the sender or receiver of IP traffic. This is critical in a wireless network since the link conditions overload the semantics of congestion without notification to any IP congestion control mechanisms. This is not as critical in the case where the link changes to higher bandwidth (higher signaling rates). It is, though, severe in the case where the link degrades to lower aggregate bandwidths. This is further exacerbated when the wireless node roves, not only within the AP coverage area, but between different APs of potentially different domains over IP mobility-aware networks.

We thus, consider essential that $\mathrm{L} 2$ rate conditioning should be propagated to the upper layers of the network. Such signal is bound to reduce reaction times of the IP (and further application) layer by informing the required peer entities (routers or hosts) about effected link status. While such L2 hint may break the model of independence between different layers of the network, they prevent the cascading effect of temporal link conditioning induced to the IP layer when transmitting over dynamically conditioned links. We argue that this is bound to have important implications on the sustainment of service quality over wireless and in particular 802.11 links.

As such, our scheme further proposes a link status notification (LSN) effected at the MAC sublayer of the $802.11 \mathrm{~b}$ protocol. The LSN signal would convey knowledge of the link's dynamic characteristics from the MAC layer to the IP layer, and signal the peer entities for the purposes of triggering some IP flow conditioning rule. We view that such notification should be quite useful further during handoff decisions.

LSN would be implemented in the driver of the wireless interface. It would further propagate as an extension on the IP header that would be interpretable by LSN-aware entities for the purposes of conditioning/adapting their IP flows.

The above scheme is currently work in progress. For it we postpone more detailed discussion and analysis as the object of a separate paper.

\section{CONCLUSIONS AND FURTHER WORK}

This paper presented a set of measurements as well as fairness-related issues of connection-less traffic over the IEEE802.11 MAC protocol vendor implementations. It has further attempted to provide with some detailed insight in the mode of operation of WLAN protocols such as the 802.11b.

This work has looked into the issue of fairness in view of the original specification requirements of maintaining connectivity as well as rate compatibility in $802.11 \mathrm{~b}$ WLAN networks. We have shown that the real performance of the HR extensions of the protocol is inhibited by the above requirements when a common acquisition sequence is adopted for all supported signaling rates. To this end, we have devised an extension to the current dynamic rate fallback scheme by enforcing some aspect of proportional fairness in bandwidth allocation effected over the discrete set of supported signaling rates for $802.11 \mathrm{~b}$. We argue that such fairness should be instigated at the lowest of the communication layers (i.e. physical) if the supported signaling rates are to provide their maximum performance.

The devised extensions allow us to argue further about a 
bandwidth-(and subsequent service-)differentiated wireless Internet. We feel that bandwidth-differentiation should not be primarily supported at the IP layer but at the MAC/PHY layer over wireless. A two-tier differentiation should be the fundamental characteristic in bandwidth provisioning over wireless, and in particular WLANs. The $802.11 \mathrm{~b}$ protocol provides an excellent candidate where such differentiation scheme can be employed. The discrete range of modulation schemes supported allow effectively the definition of discrete bandwidth allocation classes over which proportional fairness can be effected as a function of the received signal strength (RSS). Clearly, such function would manifest itself as inversely proportional to the distance between the wireless node and the base station.

We are currently devising the appropriate simulations as well as a detailed analysis of the proposed extensions over $802.11 \mathrm{~b}$ entailing link-adaptation notifications. This will provide us with results on the performance and viability of such scheme but also act as a substrate for further service qualitative considerations that may allow PCF to act in a QoS-aware fashion.

\section{ACKNOWLEDGMENTS}

This work have been sponsored by EPSRC, London, UK. We would like to thank Jon Crowcroft for their invaluable discussions and feedback during the writing of this paper. We would further like to thank Charlie Perkins and Jari Malinen from Nokia Research Center for their comments as well as the Hannu Flinck for the provision of OPNET simulation resources in NOKIAUS. We would also like to thank the UCL/MICE (aka G11) research group that has provided essential insight in aspects relative to this work. Last we would like to thank the FreeBSD community for being there and shedding light during our problem-solving endeavours.

\section{REFERENCES}

[1] V. Jacobson, "Congestion Avoidance and Control," in Proceedings of ACM SIGCOMM ' 88,1988 , pp. 314-329.

[2] J. Jubin and J. D. Tornow, "The DARPA Packet Radio Network Protocol," Proceedings of IEEE, vol. 75, pp. 21-32, 1987.

[3] S. Seshan H. Balakrishnan, V. N Padmanablhan and R. H. Katz, "A Comparison of Mechanisms for improving TCP Performance over Wireless Links," Proceeding of the ACM SIGCOMM '96, Aug. 1996.

[4] J. Kim B. Crow, I. Widjaja and P. Sakai, "IEEE 802.11 Wireless Local Area Networks," IEEE Commurications, September 1997.

[5] J. Sobrinho and A. Krishnakumar, "Real-Time Traffic ove the IEEE 802.11 Medium Access Control Layer," Bell Labs Technical Journal, 1996.

[6] P. Karn, "MACA: A new Channel Method for Packet Radio," in ARRL/CRRL Amateur Radio 9th Computer Networking Conference, 1990

[7] V. Bharghavan, "MACAW: A Media Access Protocol for Wireless LANs," in Proceedings of ACM SIGCOMM '94, Aug. 1994.

[8] C. Fullmer and J. Garcia-Luna-Aceves, "Floor Acquisition Multiple Access (FAMA) for Packet-Radio Networks," in Proceedings of ACM SIG COMM'95, 1995.

[9] M. Chuah A. DeSimone and O. Yue, "Throughput Performance of Transport-layer Protocols over Wireless LANs," in Proceedings of IEEE GLOBECOME '93, Dec. 1993.

[10] D. Bantz and F. Bauchot, "Wireless LAN Design Alternatives," IEEE Network, pp. 43-53, Apr. 1994.

[11] R. Lamaire et al., "Wireless LANs and Mobile Networking: Standards and future Directions," IEEE Communications, vol. 34, no. 8, pp. 86-94, Aug. 1996.

[12] ETS 300 652, ETSI TC-RES "Radio Equipment and Systems (RES); High Performance Radio Local Area Network (HIPERLAN); Functional Specification, ETSI, July 1995.

[13] A. Festag J. Weinmiller, M. Schalager and A. Wolisz, "Performance Study of Access Control in Wireless LANs - IEEE 802.11 DFWMAC and ETSI
RES 10 Hiperlan," in Joumal of Mobile Networks and Applications, 1997, pp. 55-67.

[14] 802.11 WG, P802.1/ Wireless LAN Medium Access Control (MAC) and Physical Layer (PHY) Specification Standard, IEEE, Oct. 1997.

[15] G. Xylomenos and G. Polyzos, "TCP and UDP Performance over a Wireless LAN"" in Proceedings of IEEE INFOCOM '99, 1999.

[16] B. Noble G. Nguyen, R. Katz and M. Satyanarayanan, "A Trace-based Approach for Modeling Wireless Channel Behaviour." in Proceeding of the Winter Simulation Conference '96, 1996.

[17] D. Duchamp and N. Reynolds, "Measured Performance of a wireless LAN," in Proceedings of the 17th IEEE Conference on Local Computer Networks, Sept. 1992

[18] T. Pagtzis, "Operational semantics and analysis of IEEE802.1 lb for connection-less IP traffic," Tech. Rep., University College London, Aug. 2000.

[19] "Rtp tools," ftp:/ftp.cs.columbia.edu/pub/schulzrine/rtptools.

[20] Y. Murayama S. Yamaguchi, "DBS: a powerful tool for TCP performance evaluation," in Proceedings of Performance and Control of Network Sys terns (SPIE'97@. Nov. 1997, vol. 3231, pp. 570-581.

[21] D. van Wykt and L. Linde, "A Turbo-coded DS/CDMA System with Embedded Walsh-Hadamard Codewords: Coder Design and Performance Evaluation" in Proceedings of 1998 IEEE 5th International Symposium on Spread Sprectrum Technuques and Applications, 1998, vol. 2. pp. 359363.

[22] M. Webster K. Halford, S. Halford and C. Andren, "Complementary Code Keying for RAKE-base Indoor Wireless Communication," in Proceedings of the 1999 IEEE Internutional Symposium on Circuits and Systems, 1999.

[23] H. Chhaya and S. Gupta, "Throughput and Fairness Properties of Asynchronous Data Transfer Metbods in the IEEE 802.11 MAC Protocol"" in Proceedings of IEEE PIMRC '95, September 1995, pp. 613-617.

[24] H. Chhaya, "Performance Evaluation of the IEEE 802.11 MAC Protocol for Wireless LANs," Msc. thesis, Graduate School of the Illinois Institute of Technology, 1996.

[25] G. Bianchi, "Performance Analysis of the IEEE 802.11 Distributed Coordination Function" Journal on Selected Areas on Communications, 2000.

[26] B. Crow et al., "Investigation of the IEEE 802.11 Medium Access Control (MAC) Sublayer Functions," in Proceedings of INFOCOM '97, 1997.

[27] "The freebsd project" http://www. freebsd.org/.

[28] L. Fratt G. Bianchi and M. Oliveri, "Performance Evaluation and Enhancement of the CSMA/CA MAC Protocol for 802.11 WLAN," in Proceedings of P'MRC' '96, 1996.

[29] A. Phonphoem A. Ganz and Z. Ganz, "Robust Superpoll Protocol for IEEE Wireless LANs," in Proceedings of MILCOM '98, 1998.

[30] C. Andren and M. Webster, "CCK Modulation Delivers 11 Mbps for High Rate IEEE 802.11 Extension," Tech. Rep., Harris Semiconductors, May 1999.

[31] C. Andren, "Short PN Sequences for Direct Sequence Spread Spectrum Radios," Tech. Rep.. Harris Semiconductors, 1997.

[32] D. Eckhardt and P. Steenkiste, "Measurement and Analysis of the Error Characteristics of an In-Building Wireless Network," in Proceedings of ACM SIGCOMM' 96 , Aug. 1996.

[33] L. Lenzini G. Anastasi, "QoS provided by the IEEE 802.11 Wireless LAN to Advanced Data Applications: a Simulation Analysis," Journal of Wireless Networks, 2000.

[34] H. Chhaya and S. Gupta, "Performance Modeling of Asynchronous Data Transfer Methods of IEEE 802.11 MAC Protocol," Joumal of Wireless Networks, pp. 217-234, 1997

[35] M. Conti L. Bononi and L. Donatiello, "Design and Performance Evaluation of a Distributed Contention Control (DCC) Mechanism for IEEE 802.11 Wireless LANs," in Proceedings of WOWMOM'98, 1998.

[36] R. van Nee et al., "New High-Rate Wireless LAN Standards," Communications Interactive, Dec. 1999.

[37] Z. Velkov and L. Gavrilovska, "Performance of the IEEE 802.11 Wireless LANs under Influence of Hidden Terminals and Pareto Distributed Packet Traffic," in Proceedings of ICPWC '99, 1999. 